\title{
Convergence in law to operator fractional Brownian motions
}

\author{
Hongshuai Dai * \\ College of Mathematics and Information Sciences, Guangxi University, \\ Nanning 530004, China.
}

October 26, 2018

\begin{abstract}
In this paper, we provide two approximations in law of operator fractional Brownian motions. One is constructed by Poisson processes, and the other generalizes a result of Taqqu (1975).
\end{abstract}

2000 Mathematics subject classification: 60F17; 60G15

Keywords: Operator fractional Brownian motion, Poisson processes, vector-valued Gaussian sequence, weak convergence.

\section{Introduction}

Self-similar processes, first studied rigorously by Lamperti [18] under the name "semi-stable", are stochastic processes that are invariant in distribution under suitable scaling of time and space. There has been an extensive literature on self-similar processes. We refer to Vervaat [30] for general properties, to Samorodnitsky and Taqqu [28] [Chaps.7 and 8] for studies on Gaussian and stable self-similar processes and random fields.

The definition of self-similarity has been extended to allow for scaling by linear operators on $\mathbb{R}^{d}$, and the corresponding processes are called operator self-similar (o.s.s) processes in the literature. See Laha and Rohatgi [19, Hudson and Mason [17, and Sato 26. Various examples of operator self-similar Gaussian and non-Gaussian processes have been constructed and studied by Maejima and Mason 21 and Mason and Xiao 22. The theory of operator self-similarity runs somewhat parallel to that of operator stable measures and is also related to that of operator scaling random fields. See, for instance, Meerschaert and Scheffler [24], Biermé et al. 2] and the references therein.

Let $\operatorname{End}\left(\mathbb{R}^{d}\right)$ be the set of linear operators on $\mathbb{R}^{d}$ (endomorphisms) and let $A u t\left(\mathbb{R}^{d}\right)$ be the set of invertible linear operators (automorphisms) in $\operatorname{End}\left(\mathbb{R}^{d}\right)$. For convenience, we will not distinguish an operator $D \in \operatorname{End}\left(\mathbb{R}^{d}\right)$ from its associated matrix relative to the standard basis of $\mathbb{R}^{d}$.

In this paper, we will use the following definition of o.s.s. processes, which corresponds to that of Sato [26, but is stronger than that of Hudson and Mason [17.

An $\mathbb{R}^{d}$ - valued stochastic process $\tilde{Y}=\{\tilde{Y}(t)\}$ is said to be operator self-similar if it is stochastically continuous, and there exists a $D \in E n d\left\{\mathbb{R}^{d}\right\}$ such that for every $c>0$

$$
\tilde{Y}(c t) \stackrel{d}{=} c^{D} \tilde{Y}(t), t \in \mathbb{R}
$$

where $\stackrel{d}{=}$ denotes equality of all finite-dimensional distributions, and

$$
\left.c^{D}=\exp ((\log c) D)\right)=\sum_{k=0}^{\infty} \frac{1}{k !}(\log c)^{k} D^{k} .
$$

*E-mail:mathdsh@gmail.com 
Any matrix for which (1.1) holds is called an exponent of the o.s.s process $\tilde{Y}$.

We say that a process $\tilde{Y}=\{\tilde{Y}(t)\}$ has stationary increments (s.i.) if for every $b \in \mathbb{R}$

$$
\tilde{Y}(\cdot+b)-\tilde{Y}(b) \stackrel{d}{=} \tilde{Y}(\cdot)-\tilde{Y}(0) .
$$

One of examples of operator self-similar processes is the operator fractional Brownian motion (OFBM). OFBMs are mean-zero, o.s.s., Gaussian processes with stationary increments. They are of interest in several areas and for reasons similar to those in the univariate case. For example, they are obtained and used in the context of multivariate time series and long range dependence (see, for example, Chung [3, Davidson and de Jong 6], Davidson and Hashimzade 7, Dolado and Marmol [12, Robinson [25, and Marinucci and Robinson [23]). They are also studied in problems related to, for example, queuing systems and large deviations (see Delgado [9], and Konstantopoulos and Lin[20]). In particular, Mason and Xiao [22 studied the sample properties of a particular class of OFBMs. Didier and Pipiras [10, 11] studied the basic properties of OFBMs, such as the time reversibility, the behavior of the spectral density around zero, and so on.

On the other hand, weak convergence to FBM processes has been studied extensively since the works of Davydov [5] and Taqqu [29. In recent years many new results on approximations of FBM processes have been established. For example, Enriquez [13] showed that a FBM can be approximated in law by appropriately normalized correlated random walks. Delgado and Jolis 8 proved that the law of a FBM can be weakly approximated by the law of some processes constructed from a standard Poisson process.

Despite a growing interest in OFBMs, there is little work that studies weak limit theorems for OFBMs. In this paper we will study weak limit theorems for OFBMs. We will extend two approximation results for the one-dimensional FBM to the multivariate case of OFBM. The rest of this paper is organized as follows. In Section 2, we recall some preliminaries and present the main results of this paper. Based on Poisson processes, we prove weak limit theorem for OFBMs in Section 3. Based on a stationary sequence, we study weak convergence to OFBMs in Section 4.

\section{Preliminaries}

We first recall some facts we need later. Throughout this paper, we will use $\|x\|_{E}$ to denote the usual Euclidean norm of $x \in \mathbb{R}^{d}$. For $A \in \operatorname{End}\left(\mathbb{R}^{d}\right)$, let $\|A\|=\max _{\|x\|_{E}=1}\|A x\|_{E}$ denote the operator norm of $A$. It is easy to see that for $A, B \in \operatorname{End}\left(\mathbb{R}^{d}\right)$,

$$
\|A B\| \leq\|A\| \cdot\|B\|
$$

and for every $A=\left(A_{i j}\right)_{d \times d} \in \operatorname{End}\left(\mathbb{R}^{d}\right)$,

$$
\max _{1 \leq i, j \leq d}\left|A_{i j}\right| \leq\|A\| \leq d^{\frac{3}{2}} \max _{1 \leq i, j \leq d}\left|A_{i j}\right| .
$$

Let $\sigma(A)$ be the collection of all eigenvalues of $A$. We denote

$$
\lambda_{A}=\min \{\operatorname{Re} \lambda: \lambda \in \sigma(A)\} \text { and } \Lambda_{A}=\max \{\operatorname{Re} \lambda: \lambda \in \sigma(A)\} .
$$

As standard for the multivariate context, we furthermore assume that the OFBM is proper. A random variable in $\mathbb{R}^{d}$ is said to be proper if the support of its distribution is not contained in a proper hyperplane of $\mathbb{R}^{d}$.

Let $x^{\prime}$ denote the transpose vector of $x \in \mathbb{R}^{d}$, and $B^{*}$ be the adjoint operator of $B$. Let $x_{+}=\max \{x, 0\}$ and $x_{-}=\max \{-x, 0\}$.

Didier and Pipiras [10] established the integral representations of OFBMs in the spectral domain. The following lemma comes from Didier and Pipiras [10][Theorem 3.1]. 
Lemma 2.1 Let $D$ be a linear operator on $\mathbb{R}^{d}$ with $0<\Lambda_{D}, \lambda_{D}<1$. Let $X=\{X(t)\}$ be an OFBM with o.s.s. exponent $D$. Then $X$ admits the integral representation

$$
X(t) \stackrel{d}{=} \int_{\mathbb{R}} \frac{e^{i t x}-1}{i x}\left(x_{+}^{-\left(D-\frac{I}{2}\right)} A+x_{-}^{-\left(D-\frac{I}{2}\right)} \bar{A}\right) W(d x)
$$

for some linear operator $A$ on $\mathbb{C}^{d}$. Here, $\bar{A}$ denotes the complex conjugate and

$$
W(x):=W_{1}(x)+i W_{2}(x)
$$

denotes a complex-valued multivariate Brownian motion such that $W_{1}(-x)=W_{1}(x)$ and $W_{2}(-x)=$ $-W_{2}(x), W_{1}(x)$ and $W_{2}(x)$ are independent, and the induced random measure $W(x)$ satisfies

$$
\mathbb{E}\left[W(d x) W^{*}(d x)\right]=d x,
$$

where $W^{*}$ is the adjoint operator of $W$.

Remark 2.1 For fixed $t \in \mathbb{R}$, let

$$
\begin{aligned}
F(x) & =\frac{e^{i t x}-1}{i x}\left(x_{+}^{-\left(D-\frac{I}{2}\right)} A+x_{-}^{-\left(D-\frac{I}{2}\right)} \bar{A}\right) \\
& =F_{1}(x)+i F_{2}(x) .
\end{aligned}
$$

Since $F(x)=\bar{F}(-x), F_{1}(x)=F_{1}(-x)$ and $F_{2}(x)=-F_{2}(-x)$.

Inspired by Samorodnitsky and Taqqu [28] [Chap. 7], up to a multiplicative constant, we can rewrite $\{X(t)\}$ as follows.

$$
X(t) \stackrel{d}{=} \int_{0}^{\infty} G_{1}(x, t) W_{1}(d x)+\int_{0}^{\infty} G_{2}(x, t) W_{2}(d x)
$$

where

$$
\begin{aligned}
& G_{1}(x, t)=\frac{\sin t x}{x} x^{-\left(D-\frac{I}{2}\right)} A_{1}+\frac{\cos t x-1}{x} x^{-\left(D-\frac{I}{2}\right)} A_{2}, \\
& G_{2}(x, t)=\frac{\sin t x}{x} x^{-\left(D-\frac{I}{2}\right)} A_{2}+\frac{1-\cos t x}{x} x^{-\left(D-\frac{I}{2}\right)} A_{1},
\end{aligned}
$$

and

$$
A=A_{1}+i A_{2}
$$

Remark 2.2 Using the same method as Mason and Xiao [22, we can get that for $i=1,2$,

$$
\int_{\mathbb{R}_{+}}\left\|G_{i}(u, t)\right\|^{2} d u<\infty
$$

In this paper, we study weak limit theorems for the OFBM $X$. We first recall some results about weak convergence to FBMs. Stroock [27] showed that

Lemma 2.2 Let $\left\{N_{n}(t), n=1,2 \cdots\right\}$ be a sequence of Poisson processes with intensity $n$, and construct the continuous processes $\left\{\tilde{Y}_{n}(t)\right\}$ by

$$
\tilde{Y}_{n}(t)=\sqrt{n} \int_{0}^{t}(-1)^{N_{n}(s)} d s, 0 \leq t \leq 1 .
$$

Then the laws of $\left\{\tilde{Y}_{n}\right\}$ converge weakly, in $\mathcal{C}([0,1])$, to the law of a Brownian motion, as $n \rightarrow \infty$. 
Using the same method as Delgado and Jolis [8], one can easily get

Corollary 2.1 Let $N_{n}(t)$ and $\hat{N}_{n}(t)$ be two independent Poisson processes with intensity $n$, and construct the processes $\left\{\hat{Y}_{n}(t)\right\}$ by

$$
\hat{Y}_{n}(t)=\sqrt{n} \int_{\mathbb{R}_{+}} \frac{(1-\cos t x)}{x^{H+1 / 2}}(-1)^{N_{n}(x)} d x+\sqrt{n} \int_{\mathbb{R}_{+}} \frac{\sin t x}{x^{H+1 / 2}}(-1)^{\hat{N}_{n}(x)} d x
$$

where $H \in(0,1)$. Then the laws of $\left\{\hat{Y}_{n}(t), n=1,2, \cdots\right\}_{t \in[0,1]}$ converge weakly in $\mathcal{C}([0,1])$, to the law of a fractional Brownian motion with index $H \in(0,1)$, as $n \rightarrow \infty$.

Inspired by Corollary 2.1, we want to show that the OFBM $X$ given by (2.5) can also be approximated by a sequence of processes similar to (2.7).

Let $\theta_{n}^{0}(t)=\sqrt{n}(-1)^{N_{n}(t)}$ and

$$
\theta_{n}(t)=\left(\theta_{n}^{1}(t), \cdots, \theta_{n}^{d}(t)\right)^{\prime}
$$

where $\theta_{n}^{i}(t), i \in\{1, \cdots, d\}$ are independent copies of $\theta_{n}^{0}(t)$. Furthermore we define

$$
\int_{0}^{t} \theta_{n}(s) d s=\left(\int_{0}^{t} \theta_{n}^{1}(s) d s, \cdots, \int_{0}^{t} \theta_{n}^{d}(s) d s\right)^{\prime} .
$$

Inspired by Corollary 2.1 and the construction of $\left\{\hat{Y}_{n}\right\}$, we define the sequence $\left\{X_{n}(t)\right\}_{n \in \mathbb{N}}$ as

$$
X_{n}(t)=\int_{0}^{\infty} G_{1}(x, t) \theta_{n}(x) d x+\int_{0}^{\infty} G_{2}(x, t) \hat{\theta}_{n}(x) d x,
$$

where $\hat{\theta}_{n}(x)$ is an independent copy of $\theta_{n}(x)$. Then

Theorem 2.1 The laws of $\left\{X_{n}(t), n=1,2, \cdots\right\}_{t \in[0,1]}$ given by (2.10) in $\mathcal{C}^{d}[0,1]$ converge weakly to the law of the OFBM $X$ given by (2.5), as $n \rightarrow \infty$, where $\mathcal{C}^{d}[0,1]=\mathcal{C}\left([0,1], \mathbb{R}^{d}\right)$.

On the other hand, Taqqu [29] showed that a FBM can be approximated in law by normalized partial sums of stationary random variables. Since OFBMs are multivariate extensions of FBMs, it is interesting to extend Taqqu's [29] result to the OFBM case.

Before we state our result, we first introduce the following notation.

$$
\{A(n)\}=\left\{\left(A_{i j}(n)\right)_{d \times d}\right\} \in \operatorname{End}\left(\mathbb{R}^{d}\right)
$$

and

$$
\{B(n)\}=\left\{\left(B_{i j}(n)\right)_{d \times d}\right\} \in \operatorname{End}\left(\mathbb{R}^{d}\right)
$$

are asymptotically equivalent, as $n \rightarrow \infty$, if for any $i, j \in\{1, \cdots, d\}$, one of the following cases holds:

(i) There exists $N_{0} \in \mathbb{N}$ such that for all $n \geq N_{0}$,

$$
B_{i j}(n) \neq 0 \text { and } \lim _{n \rightarrow \infty} A_{i j}(n) / B_{i j}(n)=1 .
$$

(ii) There exists $N_{1} \in \mathbb{N}$ such that for all $n \geq N_{1}$,

$$
B_{i j}(n)=0 \text { and } A_{i j}(n)=0 .
$$


We denote this as $A(n) \sim B(n)$, as $n \rightarrow \infty$.

Recall that a process $\tilde{Y}=\{\tilde{Y}(t)\}$ is time reversible if it satisfies

$$
\tilde{Y}(t) \stackrel{d}{=} \tilde{Y}(-t), t \in \mathbb{R} .
$$

The author refers the readers to Didier and Pipiras [10] for time reversible Gaussian processes with stationary increments.

Due to the following lemma, we only can extend Taqqu's result to the time reversible case.

Lemma 2.3 Let $\left\{\hat{Z}_{i}, i=1,2, \cdots\right\}$ be a stationary proper mean-zero Gaussian sequence of $\mathbb{R}^{d}$ valued vectors, and

$$
\hat{S}_{n}(t)=\sum_{i=1}^{\lfloor n t\rfloor} \hat{Z}_{i} .
$$

If all the finite-dimensional distributions of $\left\{\hat{S}_{n}(t)\right\}$ converge to the corresponding finite-dimensional distributions of a mean-zero proper Gaussian process $Z=\{Z(t)\}$. Then $Z$ is time reversible.

Proof: Define

$$
\mathbb{E}\left[Z(t) Z^{\prime}(s)\right]=R_{Z}(t, s)
$$

Since

$$
\hat{S}_{n}(t) \stackrel{F . D}{\Rightarrow} Z(t), \text { as } \quad n \rightarrow \infty,
$$

where $\stackrel{F . D}{\Rightarrow}$ denotes convergence of the finite-dimensional distributions, we get that

$$
\lim _{n \rightarrow \infty} \mathbb{E}\left[\hat{S}_{n}(t) \hat{S}_{n}^{\prime}(s)\right]=R_{Z}(t, s)
$$

Since $\left\{\hat{Z}_{i}\right\}$ is a stationary Gaussian sequence, we can get

$$
\mathbb{E}\left[\hat{S}_{n}(t) \hat{S}_{n}^{\prime}(s)\right]=\mathbb{E}\left[\hat{S}_{n}(s) \hat{S}_{n}^{\prime}(t)\right]
$$

It follows from (2.12) and (2.14) that

$$
R_{Z}(t, s)=R_{Z}(s, t)
$$

Since $\left\{\hat{Z}_{i}\right\}$ is a stationary Gaussian sequence, and $Z$ is a Gaussian process, we get that $Z$ has stationary increments. Therefore, by Proposition 5.1 in Didier and Pipiras [10] and (2.15), we get that the lemma holds.

Remark 2.3 It follows from Proposition 5.1 in Didier and Pipiras [10] that if the OFBM $X$ is time reversible, then

$$
\mathbb{E}\left[X(t) X^{\prime}(s)\right]=\frac{1}{2}\left[|t|^{D} \Gamma|t|^{D^{*}}+|s|^{D} \Gamma|s|^{D^{*}}-|t-s|^{D} \Gamma|t-s|^{D^{*}}\right] .
$$

Now we state our result as follows.

Theorem 2.2 Let $\left\{Z_{i}, i=1,2, \cdots\right\}$ be a stationary proper mean-zero Gaussian sequence of $\mathbb{R}^{d}$ valued vectors. We define

$$
r(i, j)=\mathbb{E}\left[Z_{i} Z_{j}^{\prime}\right]=\left(r_{k q}(|i-j|)\right)_{d \times d}
$$

Suppose that

$$
\sum_{i=1}^{N} \sum_{j=1}^{N} r(i, j) \sim K B N^{D} \Gamma N^{D^{*}} B^{*}, \text { as } N \rightarrow \infty
$$


where $\Gamma=\mathbb{E}\left[X(1) X^{\prime}(1)\right], B \in A u t\left(\mathbb{R}^{d}\right)$ and $K>0$ is a positive number. Then

$$
Q_{N}(t)=d_{N} \sum_{i=1}^{\lfloor N t\rfloor} Z_{i}
$$

with $d_{N} \sim C N^{-D} B^{-}$, converges weakly, as $N \rightarrow \infty$ in $\mathcal{D}^{d}[0,1]$, up to a multiplicative matrix from the left, to the time reversible OFBM $X$ given by (2.5) with $A_{2} A_{1}^{*}=A_{1} A_{2}^{*}$, where $C \in$ Aut $\left(\mathbb{R}^{d}\right)$, and $\mathcal{D}^{d}[0,1]=\mathcal{D}\left([0,1], \mathbb{R}^{d}\right)$.

We will prove Theorem 2.2 in Section 4 .

To end this section, we give a technical lemma which comes from Maejima and Mason [21].

Lemma 2.4 Let $D \in \operatorname{End}\left(\mathbb{R}^{d}\right)$. If $\lambda_{D}>0$ and $r>0$, then for any $\delta>0$, there exist positive constants $K_{1}$ and $K_{2}$ such that

$$
\left\|r^{D}\right\| \leq \begin{cases}K_{1} r^{\lambda_{D}-\delta}, & \text { for all } r \leq 1 \\ K_{2} r^{\Lambda_{D}+\delta}, & \text { for all } r \geq 1 .\end{cases}
$$

In the rest of this paper, most of the estimates contain unspecified constants. An unspecified positive and finite constant will be denoted by $K$, which may not be the same in each occurrence. Sometimes we shall emphasize the dependence of these constants upon parameters.

\section{Weak limit theorem based on Poisson processes}

In this section, we prove Theorem 2.1. We first state some technical results. In order to prove the main result, we need a tightness criterion for vector-valued stochastic processes.

Lemma 3.1 Let $\left\{Z_{n}(t)\right\}_{n \in \mathbb{N}}$ be a sequence of stochastic processes in $\mathcal{D}^{d}[0,1]$ satisfying:

(i) for every $n \in \mathbb{N}, Z_{n}(0)=0$ a.s.;

(ii) there exist constants $K>0, \beta>0, \alpha>1$ and an integer $N_{0} \in \mathbb{N}$ such that

$$
\mathbb{E}\left[\left\|Z_{n}(t)-Z_{n}(s)\right\|_{E}^{\beta}\right] \leq K(t-s)^{\alpha}, n \geq N_{0} .
$$

Then $\left\{Z_{n}(t)\right\}$ is tight in $\mathcal{D}^{d}[0,1]$.

The proof of Lemma 3.1 is classical (see Billingsley [1] [Chap.3] and Ethier and Kurtz [14] [Chap.3]). Here we omit the proof.

Next, we show that $\left\{X_{n}(t)\right\}$ is tight in $\mathcal{C}^{d}[0,1]$. We need the following lemmas.

Lemma 3.2 For any even $m \in \mathbb{N}$, there exists a constant $K(m, d)>0$ such that for any measurable function $f: \mathbb{R}_{+} \rightarrow \operatorname{End}\left(\mathbb{R}^{d}\right)$ with $\int_{\mathbb{R}_{+}}\|f(u)\|^{2} d u<\infty$,

$$
\mathbb{E}\left[\left\|\int_{\mathbb{R}_{+}} f(u) \theta_{n}(u) d u\right\|_{E}^{m}\right] \leq K(m, d)\left(\int_{\mathbb{R}_{+}}\|f(u)\|^{2} d u\right)^{\frac{m}{2}},
$$

where $\theta_{n}(\cdot)$ is given by (2.8).

Proof: Let $f(u)=\left(f_{i j}(u)\right)_{d \times d}=\left(f^{1}(u), \cdots, f^{d}(u)\right)^{\prime}$, where $f^{i}(u)=\left(f_{i 1}(u), \cdots, f_{i d}(u)\right)$. Then

$$
\int_{\mathbb{R}_{+}} f(u) \theta_{n}(u) d u=\left(\int_{\mathbb{R}_{+}} f^{1}(u) \theta_{n}(u) d u, \cdots, \int_{\mathbb{R}_{+}} f^{d}(u) \theta_{n}(u) d u\right)^{\prime} .
$$


It follows from (3.3) that

$$
\mathbb{E}\left[\left\|\int_{\mathbb{R}_{+}} f(u) \theta_{n}(u) d u\right\|_{E}^{m}\right] \leq K(m) \sum_{i=1}^{d} \mathbb{E}\left[\left|\int_{\mathbb{R}_{+}} f^{i}(u) \theta_{n}(u) d u\right|^{m}\right] .
$$

For each $i \in\{1, \cdots, d\}$, we have

$$
\mathbb{E}\left[\left|\int_{\mathbb{R}_{+}} f^{i}(u) \theta_{n}(u) d u\right|^{m}\right] \leq K(m) \sum_{j=1}^{d} \mathbb{E}\left[\left|\int_{\mathbb{R}_{+}} f_{i j}(u) \theta_{n}^{j}(u) d u\right|^{m}\right] .
$$

Since $\int_{\mathbb{R}_{+}}\|f(u)\|^{2} d u<\infty$, it follows from (2.2) that

$$
\int_{\mathbb{R}_{+}}\left|f_{i j}(u)\right|^{2} d u \leq \int_{\mathbb{R}_{+}}\|f(u)\|^{2} d u<\infty .
$$

Using the same method as in Delgado and Jolis 8, we have

$$
\mathbb{E}\left[\left|\int_{\mathbb{R}_{+}} f_{i j}(u) \theta_{n}^{j}(u) d u\right|\right]^{m} \leq K(m)\left(\int_{\mathbb{R}_{+}}\left|f_{i j}(u)\right|^{2} d u\right)^{\frac{m}{2}} .
$$

Combining (2.2), (3.4), (3.5) and (3.7), we get that the lemma holds.

Lemma 3.3 Choose $\delta>0$, such that $\lambda_{D}-\delta>0$ and $\Lambda_{D}+\delta<1$, then for any $0 \leq s<t \leq 1$ and even $m \in \mathbb{N}$, we have

$$
\mathbb{E}\left[\left\|X_{n}(t)-X_{n}(s)\right\|_{E}^{m}\right] \leq K(m, \delta, d)(t-s)^{m H}
$$

where $H=\lambda_{D}-\delta$.

Proof: Using the inequality $(a+b)^{m} \leq 2^{m-1}\left(a^{m}+b^{m}\right)$ for $a, b>0$, we get that there exists a constant $K(m)>0$ such that

$$
\begin{aligned}
\mathbb{E}\left[\left\|X_{n}(t)-X_{n}(s)\right\|_{E}^{m}\right] \leq & K(m) \mathbb{E}\left[\left\|\int_{\mathbb{R}_{+}}\left(G_{1}(x, t)-G_{1}(x, s)\right) \theta_{n}(x) d x\right\|_{E}^{m}\right] \\
& +K(m) \mathbb{E}\left[\left\|\int_{\mathbb{R}_{+}}\left(G_{2}(x, t)-G_{2}(x, s)\right) \hat{\theta}_{n}(x) d x\right\|_{E}^{m}\right] \\
= & K(m) I_{1}(m)+K(m) I_{2}(m),
\end{aligned}
$$

where

$$
I_{1}(m)=\mathbb{E}\left[\left\|\int_{\mathbb{R}_{+}}\left(G_{1}(x, t)-G_{1}(x, s)\right) \theta_{n}(x) d x\right\|_{E}^{m}\right]
$$

and

$$
I_{2}(m)=\mathbb{E}\left[\left\|\int_{\mathbb{R}_{+}}\left(G_{2}(x, t)-G_{2}(x, s)\right) \hat{\theta}_{n}(x) d x\right\|_{E}^{m}\right] .
$$

Since $\hat{\theta}_{n}(x)$ is an independent copy of $\theta_{n}(x)$, we only deal with $I_{1}(m) . I_{2}(m)$ can be done in the same method.

By Lemma 3.2 and Remark 2.2, we get that

$$
I_{1}(m) \leq K(m, d)\left(\int_{\mathbb{R}_{+}}\left\|G_{1}(x, t)-G_{1}(x, s)\right\|^{2} d x\right)^{\frac{m}{2}} .
$$


On the other hand,

$$
\begin{aligned}
\left\|G_{1}(x, t)-G_{1}(x, s)\right\|^{2}= & \|(\sin t x-\sin s x)\left(\frac{1}{x}\right)^{D+\frac{I}{2}} \\
& +(\cos t x-\cos s x)\left(\frac{1}{x}\right)^{D+\frac{I}{2}} \|^{2} \\
\leq K & \left\|(\sin t x-\sin s x)\left(\frac{1}{x}\right)^{D+\frac{I}{2}}\right\|^{2} \\
& +K\left\|(\cos t x-\cos s x)\left(\frac{1}{x}\right)^{D+\frac{I}{2}}\right\|^{2} .
\end{aligned}
$$

By (3.10) and (3.11),

$$
\begin{aligned}
I_{1}(m) \leq & K(m)\left[\int_{\mathbb{R}_{+}}\left[\left\|(\sin t x-\sin s x)\left(\frac{1}{x}\right)^{D+\frac{I}{2}}\right\|^{2}\right] d x\right]^{\frac{m}{2}} \\
& +K(m)\left[\int_{\mathbb{R}_{+}}\left[\left\|(\cos t x-\cos s x)\left(\frac{1}{x}\right)^{D+\frac{I}{2}}\right\|^{2}\right] d x\right]^{\frac{m}{2}} .
\end{aligned}
$$

For the sake of conciseness, we define

$$
U_{1}(m)=\left[\int_{\mathbb{R}_{+}}\left[\left\|(\sin t x-\sin s x)\left(\frac{1}{x}\right)^{D+\frac{I}{2}}\right\|^{2}\right] d x\right]^{\frac{m}{2}}
$$

and

$$
U_{2}(m)=\left[\int_{\mathbb{R}_{+}}\left[\left\|(\cos t x-\cos s x)\left(\frac{1}{x}\right)^{D+\frac{I}{2}}\right\|^{2}\right] d x\right]^{\frac{m}{2}} .
$$

It follows from Lemma 2.4 that

$$
\begin{aligned}
U_{2}(m) \leq K( & m, \delta, d)\left(\int_{0}^{1}(\cos t x-\cos s x)^{2}\left(\frac{1}{x}\right)^{2\left(\Lambda_{D}+\delta+\frac{1}{2}\right)} d x\right)^{\frac{m}{2}} \\
& +K(m, \delta, d)\left(\int_{1}^{\infty}(\cos t x-\cos s x)^{2}\left(\frac{1}{x}\right)^{2\left(\lambda_{D}-\delta+\frac{1}{2}\right)} d x\right)^{\frac{m}{2}} .
\end{aligned}
$$

The first term on the r.h.s. of (3.13) can be bounded from above by

$$
\begin{aligned}
K(m, & \delta, d)\left(\int_{0}^{1} \sin ^{2}((t-s) x / 2)\left(\frac{1}{x}\right)^{2\left(\Lambda_{D}+\delta+\frac{1}{2}\right)} d x\right)^{\frac{m}{2}} \\
& \leq K(m, \delta, d)(t-s)^{m\left(\Lambda_{D}+\delta\right)}\left(\int_{0}^{\infty} \frac{\sin ^{2} x}{x^{2\left(\Lambda_{D}+\delta+\frac{1}{2}\right)}} d x\right)^{\frac{m}{2}} \\
& \leq K(m, \delta, d)(t-s)^{m\left(\Lambda_{D}+\delta\right)}\left(\int_{0}^{1} \frac{\sin ^{2} x}{x^{2\left(\Lambda_{D}+\delta+\frac{1}{2}\right)}} d x+\int_{1}^{\infty} \frac{1}{x^{2\left(\Lambda_{D}+\delta+\frac{1}{2}\right)}} d x\right)^{\frac{m}{2}} .
\end{aligned}
$$

The second term on the r.h.s. of (3.13) can be bounded from above by

$$
\begin{aligned}
K(m, & \delta, d)\left(\int_{1}^{\infty} \sin ^{2}((t-s) x / 2)\left(\frac{1}{x}\right)^{2\left(\lambda_{D}-\delta+\frac{1}{2}\right)} d x\right)^{\frac{m}{2}} \\
& \leq K(m, \delta, d)(t-s)^{m\left(\lambda_{D}-\delta\right)}\left(\int_{0}^{\infty} \frac{\sin ^{2} x}{x^{2\left(\lambda_{D}-\delta+\frac{1}{2}\right)}} d x\right)^{\frac{m}{2}} \\
& \leq K(m, \delta, d)(t-s)^{m\left(\lambda_{D}-\delta\right)}\left(\int_{0}^{1} \frac{\sin ^{2} x}{x^{2\left(\lambda_{D}-\delta+\frac{1}{2}\right)}} d x+\int_{1}^{\infty} \frac{1}{x^{2\left(\lambda_{D}-\delta+\frac{1}{2}\right)}} d x\right)^{\frac{m}{2}} .
\end{aligned}
$$


It follows from (3.13) to (3.15) that

$$
U_{2}(m) \leq K(m, \delta, d)(t-s)^{m H},
$$

where $H=\lambda_{D}-\delta$.

Using the same method as the proof of (3.16), we get that

$$
U_{1}(m) \leq K(m, \delta, d)(t-s)^{m H} .
$$

It follows from (3.12), (3.16) and (3.17) that

$$
I_{1}(m) \leq K(m, \delta, d)(t-s)^{m H} .
$$

Using the same method as the proof of (3.18), we get

$$
I_{2}(m) \leq K(m, \delta, d)(t-s)^{m H} .
$$

The lemma follows from (3.9), (3.18) and (3.19).

Remark 3.1 It follows from Ethier and Kurtz [14] [Chap.3 Proposition 10.3] that Lemma 3.3]also shows that $\mathbb{P}\left(X_{n} \in \mathcal{C}^{d}[0,1]\right)=1$.

In the following, we will prove that $\left\{X_{n}(t), t \in[0,1]\right\}$ given by (2.10) converges weakly in $\mathcal{C}^{d}[0,1]$ to the OFBM $X$ given by (2.5), as $n \rightarrow \infty$. In order to obtain it, we need the following lemma.

Lemma 3.4 The laws of $\left\{\int_{0}^{t} \theta_{n}(u) d u\right\}$ converge weakly in $\mathcal{C}^{d}[0,1]$ to the law of an $\mathbb{R}^{d}$-valued Brownian motion, as $n \rightarrow \infty$.

Proof: For every even $m \in \mathbb{N}$ and any $0 \leq s<t \leq 1$,

$$
\mathbb{E}\left[\left\|\int_{0}^{t} \theta_{n}(u) d u-\int_{0}^{s} \theta_{n}(u) d u\right\|_{E}^{m}\right] \leq K(m) \sum_{i=1}^{d} \mathbb{E}\left[\left|\int_{s}^{t} \theta_{n}^{i}(u) d u\right|^{m}\right] .
$$

By Lemma 2 in Delgado and Jolis [8], we get

$$
\mathbb{E}\left[\left\|\int_{0}^{t} \theta_{n}(u) d u-\int_{0}^{s} \theta_{n}(u) d u\right\|_{E}^{m}\right] \leq K(m)(t-s)^{\frac{m}{2}} .
$$

By Lemma 3.1 and Proposition 10.3 in Ethier and Kurtz [14] [Chap.3], (3.21) implies the tightness of $\left\{\int_{0}^{t} \theta_{n}(u) d u\right\}$.

On the other hand, the convergence of all finite-dimensional distributions follows from Lemma 2.2 and the well-known Cramér-Wold device (Jurek and Mason [16 [Chap.1] and Whitt [31] [Chap.3]).

Finally tightness plus convergence of the finite-dimensional distributions implies the weak convergence.

The proof of Theorem 2.1 is as follows.

Proof of Theorem 2.1: We first prove the tightness of the laws of $\left\{X_{n}(t), t \in[0,1]\right\}$. From Lemma 3.3, for every even $m \in \mathbb{N}$, which satisfies $m H>1$, inequality (3.8) holds. It follows from Lemma 3.1 that $\left\{X_{n}\right\}$ is tight.

Now we proceed with the identification of the limit law. It is sufficient to prove that for any $q \in \mathbb{N}, a_{1}, \cdots, a_{q} \in \mathbb{R}$ and $t_{1} \cdots, t_{q} \in[0,1]$,

$$
\sum_{m=1}^{q} a_{m} X_{n}\left(t_{m}\right) \Rightarrow \sum_{m=1}^{q} a_{m} X\left(t_{m}\right)
$$


We define for any $m \in\{1, \cdots, q\}$,

$$
\begin{aligned}
\int_{\mathbb{R}_{+}} G_{1}\left(t_{m}, x\right) \theta_{n}(x) d x & =\left(\tilde{X}_{n}^{1}\left(t_{m}\right), \cdots, \tilde{X}_{n}^{d}\left(t_{m}\right)\right)^{\prime}, \\
\int_{\mathbb{R}_{+}} G_{2}\left(t_{m}, x\right) \hat{\theta}_{n}(x) d x & =\left(\hat{X}_{n}^{1}\left(t_{m}\right), \cdots, \hat{X}_{n}^{d}\left(t_{m}\right)\right)^{\prime},
\end{aligned}
$$

and

$$
\begin{aligned}
& \int_{\mathbb{R}_{+}} G_{1}\left(t_{m}, x\right) d W_{1}(x)=\left(\tilde{X}^{1}\left(t_{m}\right), \cdots, \tilde{X}^{d}\left(t_{m}\right)\right)^{\prime}, \\
& \int_{\mathbb{R}_{+}} G_{2}\left(t_{m}, x\right) d W_{2}(x)=\left(\hat{X}^{1}\left(t_{m}\right), \cdots, \hat{X}^{d}\left(t_{m}\right)\right)^{\prime} .
\end{aligned}
$$

By the Cramér-Wold device, in order to prove (3.22) we only need to show that for any $\left(b^{1}, \cdots, b^{d}\right)^{\prime} \in \mathbb{R}^{d}$

$$
\sum_{j=1}^{d} \sum_{m=1}^{q} a_{m} b^{j} \tilde{X}_{n}^{j}\left(t_{m}\right) \Rightarrow \sum_{j=1}^{d} \sum_{m=1}^{q} a_{m} b^{j} \tilde{X}^{j}\left(t_{m}\right),
$$

and

$$
\sum_{j=1}^{d} \sum_{m=1}^{q} a_{m} b^{j} \hat{X}_{n}^{j}\left(t_{m}\right) \Rightarrow \sum_{j=1}^{d} \sum_{m=1}^{q} a_{m} b^{j} \hat{X}^{j}\left(t_{m}\right),
$$

since $\theta_{n}(u)$ and $\hat{\theta}_{n}(u)$ are independent. Now we only prove that (3.23) holds. (3.24) can be done in the same way.

In order to simplify the notation, let

$$
W_{1}(x)=\left(W_{1}^{1}(x), W_{1}^{2}(x), \cdots, W_{1}^{d}(x)\right)^{\prime},
$$

and

$$
f(u, x)=G_{1}(u, x)=\left(f_{j i}(u, x)\right)_{d \times d}=\left(f^{1}(u, x), \cdots, f^{d}(u, x)\right)^{\prime},
$$

where $f^{j}(u, x)=\left(f_{j 1}(u, x), \cdots, f_{j d}(u, x)\right)$.

Therefore, we can rewrite (3.23) as follows.

$$
\sum_{i=1}^{d} H_{n}^{i} \Rightarrow \sum_{i=1}^{d} H^{i}
$$

where

$$
\begin{aligned}
H_{n}^{i} & =\sum_{j=1}^{d} \sum_{m=1}^{q} b^{j} a_{m} \int_{\mathbb{R}_{+}} f_{j i}\left(t_{m}, x\right) \theta_{n}^{i}(x) d x=\int_{\mathbb{R}_{+}} F_{i}(x) \theta_{n}^{i}(x) d x, \\
H^{i} & =\sum_{j=1}^{d} \sum_{m=1}^{q} b^{j} a_{m} \int_{\mathbb{R}_{+}} f_{j i}\left(t_{m}, x\right) d W_{1}^{i}(x)=\int_{\mathbb{R}_{+}} F_{i}(x) d W_{1}^{i}(x),
\end{aligned}
$$

and

$$
F_{i}(x)=\sum_{j=1}^{d} \sum_{m=1}^{q} b^{j} a_{m} f_{j i}\left(t_{m}, x\right)
$$


In order to prove (3.25), it is sufficient to prove that for any $\xi \in \mathbb{R}$,

$$
\mathbb{E}\left[\exp \left[i \xi \sum_{k=1}^{d} H_{n}^{k}\right]\right] \rightarrow \mathbb{E}\left[\exp \left[i \xi \sum_{k=1}^{d} H^{k}\right]\right]
$$

as $n \rightarrow \infty$.

Since $\theta_{n}^{k}, k=1, \cdots, d$ are mutually independent, and so are $W_{1}^{k}, k=1, \cdots, d$,

$$
\begin{aligned}
\left|\mathbb{E}\left[\exp \left[i \xi \sum_{k=1}^{d} H_{n}^{k}\right]\right]-\mathbb{E}\left[\exp \left[i \xi \sum_{k=1}^{d} H^{k}\right]\right]\right| \\
=\left|\prod_{k=1}^{d} \mathbb{E}\left[\exp \left[i \xi H_{n}^{k}\right]\right]-\prod_{k=1}^{d} \mathbb{E}\left[\exp \left[i \xi H^{k}\right]\right]\right| \\
\leq\left|\mathbb{E}\left[\exp \left[i \xi H_{n}^{1}\right]\right]-\mathbb{E}\left[\exp \left[i \xi H^{1}\right]\right]\right| \\
+\left|\prod_{k=2}^{d} \mathbb{E}\left[\exp \left[i \xi H_{n}^{k}\right]\right]-\prod_{k=2}^{d} \mathbb{E}\left[\exp \left[i \xi H^{k}\right]\right]\right|
\end{aligned}
$$

By induction on $k=2, \cdots, d$, we get that in order to prove (3.26) it is sufficient to prove that for $k \in\{1, \cdots, d\}$,

$$
H_{n}^{k} \Rightarrow H^{k}
$$

as $n \rightarrow \infty$.

By (2.2), we get that for every $k \in\{1, \cdots, d\}$,

$$
\int_{\mathbb{R}_{+}} F_{k}^{2}(u) d u \leq K \sum_{j=1}^{d} \sum_{m=1}^{q} \int_{\mathbb{R}_{+}} f_{j k}^{2}\left(u, t_{m}\right) d u<\infty .
$$

Therefore, the proof of (3.27) follows the lines of the proof of Theorem 2.1 in Dai and Li [4].

Combining (3.25) and (3.27), we get that (3.23) holds. Similarly, we get that (3.24) holds.

By Theorem 7.8 in Ethier and Kurtz [14][Chap.3] and Remark 3.1, we get that Theorem 2.1] holds. The proof is done.

\section{Weak convergence based on a stationary sequence}

In this section, we will prove Theorem 2.2. We first show that $\left\{Q_{N}(t): t \in[0,1]\right\}$ is tight in $\mathcal{D}^{d}[0,1]$. Before we prove tightness, we give a technical lemma.

Lemma 4.1 Let $\{A(n)\},\{B(n)\},\{C(n)\}$ and $\{D(n)\} \in \operatorname{End}\left\{\mathbb{R}^{d}\right\}$. If

$$
A(n) \sim C(n) \text {, as } n \rightarrow \infty,
$$

and

$$
B(n) \sim D(n) \text {, as } n \rightarrow \infty,
$$

then

$$
A(n) B(n) \sim C(n) D(n)
$$

as $n \rightarrow \infty$.

One can easily get that the lemma holds. Here we omit the proof. 
Lemma 4.2 For any $0 \leq s<t \leq 1$ and even $m \in \mathbb{N}$, there exists $N_{1} \in \mathbb{N}$ such that

$$
\mathbb{E}\left[\left\|Q_{N}(t)-Q_{N}(s)\right\|_{E}^{m}\right] \leq K(t-s)^{m\left(\lambda_{D}-\delta\right)}, \quad N \geq N_{1},
$$

where $\delta>0$ with $\lambda_{D}-\delta>0$, and $Q_{N}(t)$ is given by 2.17).

Proof: For any $s \leq t \in[0,1]$, we have

$$
\mathbb{E}\left[\left\|Q_{N}(t)-Q_{N}(s)\right\|_{E}^{m}\right]=\mathbb{E}\left[\left\|d_{N} \sum_{i=1}^{\lfloor N(t-s)\rfloor} Z_{i}\right\|_{E}^{m}\right]=\mathbb{E}\left[\left\|Q_{N}(t-s)\right\|_{E}^{m}\right],
$$

since $\left\{Z_{i}\right\}$ is a stationary sequence.

In order to simplify the notation, let

$$
d_{N} \sum_{i=1}^{\lfloor N(t-s)\rfloor} Z_{i}=\left(Q_{\lfloor N(t-s)\rfloor}^{1}, \cdots, Q_{\lfloor N(t-s)\rfloor}^{d}\right)^{\prime} .
$$

Since $(a+b)^{m} \leq 2^{m-1}\left(a^{m}+b^{m}\right)$ for $a, b>0$, we get that

$$
\mathbb{E}\left[\left\|Q_{N}(t)-Q_{N}(s)\right\|_{E}^{m}\right] \leq K(m) \sum_{i=1}^{d} \mathbb{E}\left[\left|Q_{\lfloor N(t-s)\rfloor}^{i}\right|^{m}\right] .
$$

Since $\left\{Z_{i}\right\}$ is an $\mathbb{R}^{d}$-valued Gaussian sequence, $Q_{\lfloor N(t-s)\rfloor}^{i}$ is Gaussian. So, we get that $\mathbb{E}\left[\left|Q_{\lfloor N(t-s)\rfloor}^{i}\right|^{m}\right]$ is proportional to $\left(\mathbb{E}\left[\left|Q_{\lfloor N(t-s)\rfloor}^{i}\right|^{2}\right]\right)^{\frac{m}{2}}$.

It follows from (4.3) that

$$
\mathbb{E}\left[\left\|Q_{N}(t)-Q_{N}(s)\right\|_{E}^{m}\right] \leq K\left(\mathbb{E}\left[\left\|Q_{N}(t)-Q_{N}(s)\right\|_{E}^{2}\right]\right)^{\frac{m}{2}} .
$$

Note that for an $\mathbb{R}^{d}$-valued random variable $Q=\left(Q^{1}, \cdots, Q^{d}\right)^{\prime}, \mathbb{E}\left[\|Q\|_{E}^{2}\right]$ equals the sum of diagonal entries of the correlation matrix. So it follows from (2.2) that

$$
\mathbb{E}\left[\left\|Q_{N}(t)-Q_{N}(s)\right\|_{E}^{m}\right] \leq K\left\|\mathbb{E}\left[\left[Q_{N}(t)-Q_{N}(s)\right]\left[Q_{N}(t)-Q_{N}(s)\right]^{\prime}\right]\right\|^{\|^{\frac{m}{2}}} .
$$

By (2.16) and Proposition 2.2.2 in Meerschaert and Scheffler [24],

$$
\lim _{N \rightarrow \infty} \mathbb{E}\left[Q_{N}(t)-Q_{N}(s)\right]\left[Q_{N}(t)-Q_{N}(s)\right]^{\prime}=K C(t-s)^{D} \Gamma(t-s)^{D^{*}} C^{*} .
$$

It follows from (4.5) and (4.6) that there exists $N_{1} \in \mathbb{N}$ such that for all $N \geq N_{1}$,

$$
\mathbb{E}\left[\left\|Q_{N}(t)-Q_{N}(s)\right\|_{E}^{m}\right] \leq K\left\|(t-s)^{D} \Gamma(t-s)^{D^{*}}\right\|^{\frac{m}{2}}
$$

since $\|\cdot\|$ is continuous.

By (4.7) and Lemma 2.4, we get that for all $N \geq N_{1}$,

$$
\begin{aligned}
\mathbb{E}\left[\left\|Q_{N}(t)-Q_{N}(s)\right\|_{E}^{m}\right] & \leq K\left\|(t-s)^{D}\right\|^{\frac{m}{2}} \times\left\|(t-s)^{D^{*}}\right\|^{\frac{m}{2}} \\
& \leq K(t-s)^{m\left(\lambda_{D}-\delta\right)} .
\end{aligned}
$$


The proof is completed.

Now we prove Theorem 2.2 .

Proof of Theorem 2.2. It follows from Lemmas 3.1 and 4.2 that $\left\{Q_{N}(t)\right\}$ is tight.

Now we prove the convergence of all finite-dimensional distributions. For any $p \in \mathbb{N}$ and $t_{1}, \cdots, t_{p} \in[0,1], Q_{N}\left(t_{1}\right), \cdots, Q_{N}\left(t_{p}\right)$ are jointly Gaussian, since $\left\{Z_{i}\right\}$ is a Gaussian sequence.

Define:

$$
R_{N}\left(t_{i}, t_{j}\right)=\mathbb{E}\left[Q_{N}\left(t_{i}\right) Q_{N}^{\prime}\left(t_{j}\right)\right]
$$

In order to simplify the notation, we define

$$
S_{N}(t)=\sum_{i=1}^{\lfloor N t\rfloor} Z_{i}
$$

Since $\left\{Z_{i}\right\}$ is a stationary Gaussian sequence, we have that if $t_{i}>t_{j}$,

$$
\begin{aligned}
\mathbb{E}\left[Q_{N}\left(t_{i}\right) Q_{N}^{\prime}\left(t_{j}\right)\right]= & \frac{1}{2} d_{N}\left[\mathbb{E}\left[S_{N}\left(t_{i}\right) S_{N}^{\prime}\left(t_{i}\right)\right]\right. \\
& \left.+\mathbb{E}\left[S_{N}\left(t_{j}\right) S_{N}^{\prime}\left(t_{j}\right)\right]-\mathbb{E}\left[S_{N}\left(t_{i}-t_{j}\right) S_{N}^{\prime}\left(t_{i}-t_{j}\right)\right]\right] d_{N}^{*} .
\end{aligned}
$$

By (2.16),

$$
\mathbb{E}\left[S_{N}\left(t_{j}\right) S_{N}^{\prime}\left(t_{j}\right)\right]=\sum_{i=1}^{\left\lfloor N t_{j}\right\rfloor} \sum_{k=1}^{\left\lfloor N t_{j}\right\rfloor} r(i, k) \sim K B N^{D} t_{j}^{D} \Gamma t_{j}^{D^{*}} N^{D^{*}} B^{*},
$$

as $N \rightarrow \infty$.

So, we get

$$
\lim _{N \rightarrow \infty} R_{N}\left(t_{i}, t_{j}\right)=R\left(t_{i}, t_{j}\right)
$$

where $R\left(t_{i}, t_{j}\right)=\frac{K}{2} C\left[\left|t_{i}\right|^{D} \Gamma\left|t_{i}\right|^{D^{*}}+\left|t_{j}\right|^{D} \Gamma\left|t_{j}\right|^{D^{*}}-\left|t_{i}-t_{j}\right|^{D} \Gamma\left|t_{i}-t_{j}\right|^{D^{*}}\right] C^{*}$.

It follows from Remark 2.3 that if $X$ is a time reversible OFBM, then

$$
\mathbb{E}\left[C X(t)(C X(s))^{\prime}\right]=\frac{1}{2} C\left[|t|^{D} \Gamma|t|^{D^{*}}+|s|^{D} \Gamma|s|^{D^{*}}-|t-s|^{D} \Gamma|t-s|^{D^{*}}\right] C^{*} .
$$

Since $Q_{N}\left(t_{1}\right), \cdots, Q_{N}\left(t_{p}\right)$ are jointly Gaussian, it follows from (4.11) and (4.12) that

$$
\left(Q_{N}\left(t_{1}\right), \cdots, Q_{N}\left(t_{p}\right)\right) \Rightarrow \sqrt{K}\left(C X\left(t_{1}\right), \cdots, C X\left(t_{p}\right)\right) .
$$

By Theorem 7.8 in Ethier and Kurtz [14][Chap.3], we get that the convergence of finite-dimensional distributions and tightness ensure weak convergence of $\left\{Q_{N}(t)\right\}$. The proof is completed.

At the end of this paper, we consider the OFBM given by Mason and Xiao [22]. Mason and Xiao [22] introduced the OFBM $Y=\{Y(t)\}$ as follows.

Definition 4.1 Let $D$ be a linear operator on $\mathbb{R}^{d}$ with $0<\lambda_{D}, \Lambda_{D}<1$. For any $t \in \mathbb{R}$, define

$$
Y(t)=\int_{\mathbb{R}_{+}}(1-\cos (t x))\left(\frac{1}{x}\right)^{D+I / 2} d \tilde{W}(x)+\int_{\mathbb{R}_{+}} \sin t x \cdot\left(\frac{1}{x}\right)^{D+I / 2} d \hat{W}(x),
$$

where $\tilde{W}$ is a vector-valued Gaussian measure, and $\hat{W}$ is an independent copy of $\tilde{W}$. 
By (2.4) and (4.13), we see that, up to a multiplicative constant, the OFBM $Y$ and the OFBM $X$ with $A=I$ have equal distributions.

Define

$$
Y_{n}(t)=\int_{\mathbb{R}_{+}}(1-\cos (t x))\left(\frac{1}{x}\right)^{D+I / 2} \theta_{n}(x) d x+\int_{\mathbb{R}_{+}} \sin t x \cdot\left(\frac{1}{x}\right)^{D+I / 2} \hat{\theta}_{n}(x) d x,
$$

where $\theta_{n}(x)$ is given by (2.8) and $\hat{\theta}_{n}(x)$ is an independent copy of $\theta_{n}(x)$. We immediately have

Corollary 4.1 The laws of $\left\{Y_{n}(t), t \in[0,1]\right\}$ converge weakly to the law of $Y$ given by 4.13) in $\mathcal{C}^{d}[0,1]$, as $n \rightarrow \infty$.

By (4.13), we get that

$$
\mathbb{E}\left[Y(t) Y^{\prime}(s)\right]=\frac{1}{2}\left[|t|^{D} \tilde{\Gamma}|t|^{D^{*}}+|s|^{D} \tilde{\Gamma}|s|^{D^{*}}-|t-s|^{D} \tilde{\Gamma}|t-s|^{D^{*}}\right],
$$

where $\tilde{\Gamma}=\mathbb{E}\left[Y(1) Y^{\prime}(1)\right]$. One can get that $\mathbb{E}\left[Y(t) Y^{\prime}(s)\right]=\mathbb{E}\left[Y(s) Y^{\prime}(t)\right]$. Therefore, by Proposition 5.1 in Didier and Pipiras [10], the OFBM $Y$ given by (4.13) is time reversible. Therefore, from Theorem 2.2, we get that

Corollary 4.2 Let $\left\{\tilde{Z}_{i}, i=1,2, \cdots\right\}$ be a stationary proper mean-zero Gaussian sequence of $\mathbb{R}^{d}$ valued vectors. We define

$$
\tilde{r}(i, j)=\mathbb{E}\left[\tilde{Z}_{i} \tilde{Z}_{j}^{\prime}\right]=\left(\tilde{r}_{k q}(|i-j|)\right)_{d \times d} .
$$

Suppose that

$$
\sum_{i=1}^{N} \sum_{j=1}^{N} \tilde{r}(i, j) \sim K B N^{D} \tilde{\Gamma} N^{D^{*}} B^{*}, \text { as } N \rightarrow \infty
$$

where $B \in A u t\left(\mathbb{R}^{d}\right)$ and $K>0$ is a positive number. Then

$$
\tilde{Q}_{N}(t)=d_{N} \sum_{i=1}^{\lfloor N t\rfloor} \tilde{Z}_{i},
$$

with $d_{N} \sim C N^{-D} B^{-1}$, converges weakly as $N \rightarrow \infty$ in $\mathcal{D}^{d}[0,1]$, up to a multiplicative matrix from the left, to the OFBM Y defined by (4.13).

In the following, we give a special case where conditions in Theorem 2.2 and Corollary 4.2 are satisfied.

Corollary 4.3 Suppose that $D \in A u t\left(\mathbb{R}^{d}\right)$ is a real diagonal matrix with positive diagonal entries $\lambda_{1}, \cdots, \lambda_{d}$. Let $\left\{Z_{i}, i=1,2, \cdots\right\}$ be a stationary proper mean-zero Gaussian sequence of $\mathbb{R}^{d}$-valued vectors. We define $r(|i-j|)=\mathbb{E}\left[Z_{j} Z_{i}^{\prime}\right]$. Suppose that

(i) Case $\frac{1}{2}<\lambda_{D}<1$ :

$$
r(j) \sim K \tilde{\Gamma} j^{2 D-2 I} \text { as } j \rightarrow \infty \text { with } K>0 .
$$

(ii) Case $0<\Lambda_{D}<\frac{1}{2}$ :

$$
r(j) \sim K \tilde{\Gamma} j^{2 D-2 I} \text { as } j \rightarrow \infty \text { with } K<0,
$$

and

$$
r(0)+2 \sum_{j=0}^{\infty} r(j)=\mathbb{O},
$$

where $(\mathbb{O}$ denotes the zero matrix. 
Then

$$
Q_{N}(t)=d_{N} \sum_{i=1}^{\lfloor N t\rfloor} Z_{i}
$$

with $d_{N} \sim N^{-D} C^{-1}$, converges weakly as $N \rightarrow \infty$, up to a multiplicative constant, to the OFBM $Y$ given by (4.13), where $C \in A u t\left(\mathbb{R}^{d}\right)$ is a diagonal matrix with diagonal entries

$$
\frac{1}{\sqrt{\left|2 \lambda_{1}-1\right| 2 \lambda_{1}}}, \cdots, \frac{1}{\sqrt{\left|2 \lambda_{d}-1\right| 2 \lambda_{d}}} .
$$

Proof: We will proceed to prove this corollary in two steps.

Step 1. We first deal with the case that $\frac{1}{2}<\lambda_{D}<1$. Since $D$ is a diagonal matrix, we get that $j^{2 D-2 I}$ is still a diagonal matrix. By some simple calculations, we get that the diagonal entries of $j^{2 D-2 I}$ are

$$
j^{2 \lambda_{1}-2}, \cdots, j^{2 \lambda_{d}-2} \text {. }
$$

For every $i \in\{1, \cdots, d\}$, we get from Feller [15][p.281] and Taqqu [29] that

$$
\sum_{k=1}^{j} k^{2 \lambda_{i}-2} \sim\left(2 \lambda_{i}-1\right)^{-1} j^{2 \lambda_{i}-1}, \quad \text { as } \quad j \rightarrow \infty,
$$

since $\frac{1}{2}<\lambda_{D} \leq \Lambda_{D}<1$.

So, by (4.21),

$$
\sum_{k=1}^{j} k^{2 D-2 I} \sim C_{0} j^{2 D-I}, \quad \text { as } \quad j \rightarrow \infty,
$$

where $C_{0} \in \operatorname{Aut}\left(\mathbb{R}^{d}\right)$ is a diagonal matrix with diagonal entries

$$
\left(2 \lambda_{1}-1\right)^{-1}, \cdots,\left(2 \lambda_{d}-1\right)^{-1} .
$$

On the other hand, we have

$$
E_{N}=\sum_{i=1}^{N} \sum_{j=1}^{N} r(i, j)=r(0)+\sum_{j=1}^{N-1}\left\{r(0)+2 \sum_{i=1}^{j} r(i)\right\} .
$$

Since $D$ is a diagonal matrix, $\tilde{\Gamma}$ is a diagonal matrix. Hence, we get from (4.17), (4.22) and (4.23) that, as $N \rightarrow \infty$,

$$
E_{N} \sim 2 K C_{0} \tilde{\Gamma} \sum_{j=1}^{N} j^{2 D-I} \sim 2 K C_{0} C_{1} \tilde{\Gamma} N^{2 D},
$$

where $C_{1} \in A u t\left(\mathbb{R}^{d}\right)$ is a diagonal matrix with diagonal entries

$$
\left(2 \lambda_{1}\right)^{-1}, \cdots,\left(2 \lambda_{d}\right)^{-1} .
$$

Since $C_{0}$ and $C_{1}$ are all diagonal matrices, we choose a diagonal matrix $C \in A u t\left(\mathbb{R}^{d}\right)$ such that $C^{2}=C_{0} C_{1}$. Since $C, N^{D}$ and $\tilde{\Gamma}$ are all diagonal matrices,

$$
E_{N} \sim 2 K C N^{D} \tilde{\Gamma} N^{D^{*}} C^{*} .
$$

Using the same method as the proof of Theorem 2.2, we get that $\left\{Q_{N}(t)\right\}$ converges weakly, up to a multiplicative constant, to $Y$, as $N \rightarrow \infty$. 
Step 2. We deal with the case that $0<\Lambda_{D}<\frac{1}{2}$. Corresponding to (4.18), we assume that $K<0$ in the following. For every $i \in\{1, \cdots, d\}$, we get from Feller [15][p.281] and Taqqu [29] that

$$
\sum_{k=j}^{\infty} k^{2 \lambda_{i}-2} \sim-\left(2 \lambda_{i}-1\right)^{-1} j^{2 \lambda_{i}-1}, \quad \text { as } \quad j \rightarrow \infty,
$$

since $0<\Lambda_{D}<\frac{1}{2}$. Then by (4.26),

$$
\sum_{k=j}^{\infty} k^{2 D-2 I} \sim \hat{C}_{0} j^{2 D-I}, \quad \text { as } \quad j \rightarrow \infty,
$$

where $\hat{C}_{0} \in A u t\left(\mathbb{R}^{d}\right)$ is a diagonal matrix with diagonal entries

$$
-\left(2 \lambda_{1}-1\right)^{-1}, \cdots,-\left(2 \lambda_{d}-1\right)^{-1} .
$$

On the other hand, it follows from (4.19) that

$$
r(0)+2 \sum_{i=1}^{j} r(i)=-2 \sum_{i=j+1}^{\infty} r(i) .
$$

By (4.18), (4.27) and (4.28),

$$
r(0)+2 \sum_{i=1}^{j} r(i) \sim-2 K \hat{C}_{0} \tilde{\Gamma} j^{2 D-I} .
$$

By (4.21), (4.23), (4.26) and (4.29), we get that as $N \rightarrow \infty$,

$$
E_{N} \sim-2 K \hat{C}_{0} \tilde{\Gamma} \sum_{j=1}^{N} j^{2 D-I} \sim-2 K \hat{C}_{0} C_{1} \tilde{\Gamma} N^{2 D} .
$$

Since both $\hat{C}_{0}$ and $C_{1}$ are diagonal matrices, we choose $\hat{C} \in A u t\left(\mathbb{R}^{d}\right)$ such that $(\hat{C})^{2}=\hat{C}_{0} C_{1}$. Therefore

$$
E_{N} \sim-2 K \hat{C} N^{D} \tilde{\Gamma} N^{D^{*}} \hat{C}^{*}
$$

as $N \rightarrow \infty$. Using the same method as the proof of Theorem 2.2. we get that $\left\{Q_{N}(t)\right\}$ converges weakly, up to a multiplicative constant, to $Y$, as $N \rightarrow \infty$. The proof has been completed.

Acknowledgements The author thanks Professor Yimin Xiao, Michigan State University, U.S.A., and Professor Yuqiang Li, East China Normal University, China, for stimulating discussions. I also would like to thank the reviewer for helpful comments to improve this work. This work was supported by the Scientific Research Foundation of Guangxi University (NO: XBZ110398).

\section{References}

[1] Billingsley, P., 1968. Convergence of Probability Measures. John Wiley and Sons, New York.

[2] Biermé, H., Meerschaert, M. M., Scheffler, H. P., 2007. Operator scaling stable random fields. Stoch. Process. Appl. 117, 312-332.

[3] Chung, C. F., 2002. Sample means, sample autocovariances, and linear regression of stationary multivariate long memory processes. Econometric Theory 18, 51-78. 
[4] Dai, H., Li, Y., 2010. A weak limit theorem for generalized multifractional Brownian motion. Stat. Prob. Lett. 80, 348-356.

[5] Davydov, Y., 1970. The invariance principle for stationary processes. Teor. Verojatnost. i Primenen. 15, 498-509.

[6] Davidson, J., de Jong, R. M., 2000. The functional central limit theorem and weak convergence to stochastic integrals II. Econometric Theory 16, 643-666.

[7] Davidson, J., Hashimzade, N., 2008. Alternative frequency and time domain versions of fractional Brownian motion. Econometric Theory 24, 256-293.

[8] Delgado, R., Jolis, M., 2000. Weak approximation for a class of Gaussian process. J. Appl. Probab. 37, 400-407.

[9] Delgado, R., 2007. A reflected fBm limit for fluid models with ON/OFF sources under heavy traffic. Stoch. Process. Appl. 117, 188-201.

[10] Didier, G., Pipiras, V., 2011. Integral representations and properties of operator fractional Brownian motions. Bernoulli 17,1-33.

[11] Didier, G., Pipiras, V., 2011. Exponents, symmetry groups and classification of operator fractional Brownian motions. J. Theor. Probab. to appear.

[12] Dolado, J., Marmol, F., 2004. Asymptotic inference results for multivariate long-memory processes. Econometrics Journal 7, 168-190.

[13] Enriquez, N., 2004. A simple construction of the fractional Brownian motion. Stoch. Process. Appl. 109, 203-223.

[14] Ethier, S., Kurtz, T., 1986. Markov Processes: Characterization and Convergence. John Wiley and Sons, New York.

[15] Feller, W., 1971. An Introduction to Probability Theory and its Applications (2nd). Wiley, New York.

[16] Jurek, Z. J., Mason, J. D.,1993. Operator Limit Distributions in Probability Theory. John Wiley and Sons, New York.

[17] Hudson, W.N., Mason, J. D., 1982. Operator-self-similar processes in a finite-dimensional space. Trans. Amer. Math. Soc. 273, 281-297.

[18] Lamperti, L., 1962. Semi-stable stochastic processes. Trans. Amer. Math. Soc. 104, 62-78.

[19] Laha, T.L., Rohatgi, V.K., 1982. Operator self-similar processes in $\mathbb{R}^{d}$. Stoch. Process. Appl. 12, 73-84.

[20] Konstantopoulos, T., Lin, S. J., 1996. Fractional Brownian approximations of queuing networks. In: Stochastic Networks. Lecture Notes in Statistics 117, Springer, New York, pp.257-273.

[21] Maejima, M., Mason, J. D., 1994. Operator -self-similar stable processes. Stoch. Process. Appl. 54, 139-163.

[22] Mason, J. D., Xiao, Y., 2002. Sample path properties of operator-self-similar Gaussian random fields. Theory Probab. Appl. 46, 58-78.

[23] Marinucci, D., Robinson, P., 2000. Weak convergence of multivariate fractional processes. Stoch. Process. Appl.86, 103-120. 
[24] Meerschaert, M. M., Scheffler, H. P., 2001. Limit Distributions for Sums of Independent Random Vectors: Heavy Tails in Theory and Practice. John Wiley and Sons, New York.

[25] Robinson, P., 2008. Multiple local whittle estimation in stationary systems. Annals of Statistics 36, 2508-2530.

[26] Sato, K., 1991. Self-similar processes with independent increments. Probab. Th. Rel. Fields 89, 285-300.

[27] Stroock, D., 1982. Topics in Stochastic Differential Equations. Tata Institute of Fundamental Research, Bomaby, Springer-Verlag.

[28] Samorodnitsky, G., Taqqu, M. S., 1994. Stable Non-Gaussian Random Processes: Stochastic Models with Infinite Variance. Chapman and Hall, New York, London.

[29] Taqqu, M. S., 1975. Weak convergence to fractional Brownian motion and to the Rosenblatt process. Z. Wahrsch. Verw. Gebiete 31, 287-302.

[30] Vervaat, W., 1985. Sample path properties of self-similar processes with stationary incrments. The Annals of Probability 13, 1-27.

[31] Whitt, W., 2002. Stochastic-Process Limits: An Introduction to Stochastic-Process Limits and their Application to Queues. Springer, New York. 